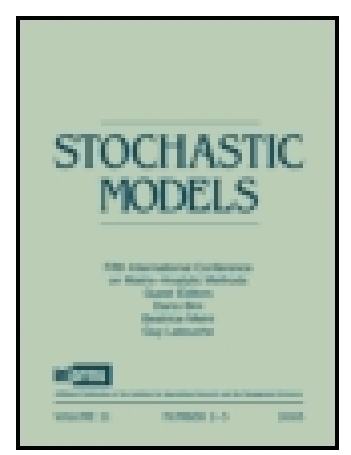

Stochastic Models

ISSN: 1532-6349 (Print) 1532-4214 (Online) Journal homepage: http://www.tandfonline.com/loi/lstm20

\title{
Solving Multi-Regime Feedback Fluid Queues
}

\section{H. Emre Kankaya \& Nail Akar}

To cite this article: H. Emre Kankaya \& Nail Akar (2008) Solving Multi-Regime Feedback Fluid Queues, Stochastic Models, 24:3, 425-450, DOI: 10.1080/15326340802232285

To link to this article: http://dx.doi.org/10.1080/15326340802232285

\section{曲 Published online: 05 Aug 2008.}

Submit your article to this journal $\pi$

\section{Џll Article views: 105}

Q View related articles $\square$

4 Citing articles: 12 View citing articles 준 


\title{
SOLVING MULTI-REGIME FEEDBACK FLUID QUEUES
}

\author{
H. Emre Kankaya and Nail Akar \\ Electrical and Electronics Engineering Department, Bilkent University, \\ Ankara, Turkey
}

\begin{abstract}
$\square \quad$ In this paper, we study Markov fluid queues with multiple thresholds, or the so-called multiregime feedback fluid queues. The boundary conditions are derived in terms of joint densities and for a relatively wide range of state types including repulsive and zero drift states. The ordered Schur factorization is used as a numerical engine to find the steady-state distribution of the system. The proposed method is numerically stable and accurate solution for problems with two regimes and $2^{10}$ states is possible using this approach. We present numerical examples to justify the stability and validate the effectiveness of the proposed approach.
\end{abstract}

Keywords Feedback queues; Markov fluid queues; Schur decomposition.

Mathematics Subject Classification Primary 60K25, 90B22; Secondary 65F15, 68M20.

\section{INTRODUCTION}

Markov fluid queues are described by a joint Markovian process $\{C(t), M(t) ; t \geq 0\}$ where $\{C(t) ; t \geq 0\}$ refers to the buffer content process and $\{M(t) ; t \geq 0\}$ is an underlying continuous-time Markov chain that determines the net rate (entry rate minus exit rate or drift) at which the buffer content $C(t)$ changes. The latter process $\{M(t) ; t \geq 0\}$ is often called the background or the modulating process of the Markov fluid queue. A key reference on Markov fluid queues is the spectral approach of Anick et al. ${ }^{[1]}$ for infinite buffer capacities. Tucker ${ }^{[2]}$ extends this analysis to finite fluid queues using the spectral approach. Kulkarni ${ }^{[3]}$ gives a more recent and extensive overview of Markov fluid queues and the spectral approach. Ramaswami $^{[4]}$ provides a systematic approach to Markov fluid queues using the matrix geometric approach. A similar method was proposed by

Received June 2007; Accepted December 2007

Address correspondence to Nail Akar, Electrical and Electronics Engineering Department, Bilkent University, Ankara, Turkey; E-mail: akar@ee.bilkent.edu.tr 
Soares and Latouche ${ }^{[5]}$ for finite buffered fluid queues. Other alternative techniques to solve Markov fluid queues include the probabilistic analysis of Asmussen ${ }^{[6]}$ using an iterative technique, the Wiener-Hopf factorization method of Rogers ${ }^{[7]}$, and the forward-backward decomposition approach of Akar and Sohraby ${ }^{[8]}$ using the matrix sign function.

More general models, known as feedback fluid queues, were introduced by Adan et al. ${ }^{[9]}$ and Scheinhardt ${ }^{[10]}$, where both the rate of change of the buffer content and the background process are allowed to depend on the instantaneous queue occupancy. We note that feedback fluid queues have proven useful for modeling controlled telecommunication networks. In one of earlier works, Elwalid and Mitra ${ }^{[1]}$ allow the drifts to change with respect to only the queue occupancy as a sub-case of feedback fluid queues and in a piecewise constant fashion, in the context of modeling a networking system with loss priorities. A similar system with drifts being piecewise continuous functions of the buffer content is analyzed by Kella and Stadje ${ }^{[12]}$. The more general feedback fluid queues in which the background process is also allowed to vary with respect to buffer content are studied by Scheinhardt et al. ${ }^{[13]}$ and Mandjes et al. ${ }^{[14]}$ (see also Mandjes et al. $^{[15]}$ ); the former study allows continuous feedback whereas the latter assumes piecewise constant feedback to model a network access system. Boxma et al. ${ }^{[16]}$ studies a model which can be viewed as a continuous feedback fluid queue with two background states and unlimited buffer content. Although continuous feedback fluid queues are powerful for modeling purposes, it is generally hard to obtain numerical results except for special cases; see for example the two-state example given by Scheinhardt et al. ${ }^{[13]}$. For the piecewise constant feedback case, Mandjes et al. ${ }^{[14]}$ propose a spectral expansion approach to solve the fluid queue of interest. However, it is not only hard to obtain numerical results for large state spaces, as stated in ${ }^{[14]}$, but also the spectral expansion approach for fluid queues turns out to be ill-conditioned, as stated by Akar and Sohraby ${ }^{[8]}$. The goal of this study is to obtain a computationally stable numerical algorithm for the steady-state solution of feedback fluid queues in case drifts and the background process are piecewise constant functions of the buffer content. In this study, we seek a method that allows us to tackle problems with relatively larger state spaces than the ones studied in the literature.

For the purpose of clarifying the contributions of the current paper, we provide a five-state feedback Markov fluid queue example in Figure 1. The buffer capacity is denoted by $B$ and the buffer content ranges in the closed interval $[0, B]$ in this example. The points 0 and $B$ are the terminal boundary points of the fluid queue. Any other point at which the infinitesimal generator of the background process or at least one of the drifts in a state change is called an intermediate boundary 


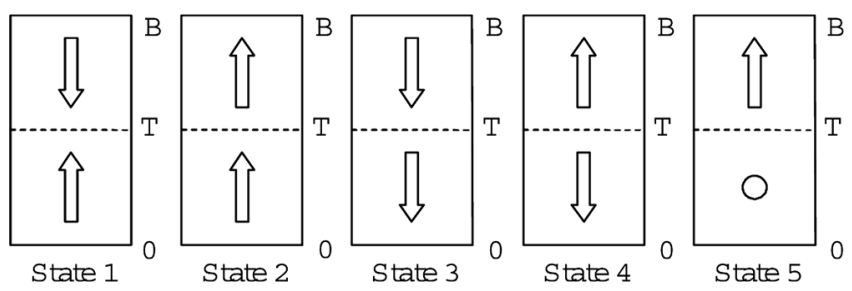

FIGURE 1 A five-state multi-regime feedback fluid queue.

point; $T$ is the only intermediate boundary point of the feedback queue in this example. The intervals between two neighboring boundary points are called regimes and the drifts and the background process are fixed throughout a regime. The example in Figure 1 has two regimes $(0, T)$ and $(T, B)$. The term multi-regime refers to a queue with multiple regimes and we will use the term multi-regime feedback fluid queue throughout the paper to refer to one in which the drifts and the infinitesimal generator of the background process are piecewise constant functions of buffer content. Drifts within a regime can take arbitrary values; zero, positive, or negative. If the drift is zero in one of the states in a given regime then the regime is said to contain a zero drift state. The arrows in Figure 1 represent the sign of the drift; for example the drift is positive in both regimes for the second state. Moreover, a circle indicates a zero drift; for example the drift is zero for the fifth state in the lower regime. We now classify the boundary points according to the drift values just at those boundary points and the neighboring regimes for each state. An intermediate boundary point is said to be absorbing for a given state if the drift at that state is negative in the regime above and it is positive in the regime below. We assume that for an absorbing state, the drift at the corresponding boundary point is zero. In Figure 1, state 1 is an absorbing state at boundary point $T$. Next, we consider intermediate boundary points at which the drifts in the neighboring regimes are both nonzero and have the same sign for a given state. In this case, we assume that the drift at the boundary point possesses the same sign for the given state. We call such states emitting at the corresponding boundary point; states 2 and 3 are emitting states at the intermediate boundary $T$. Finally, if the drift for a given state is positive in the regime above and it is negative in the regime below an intermediate boundary point, then this boundary point is said to be repulsive for that state; for example, boundary point $T$ is repulsive for state 4 . For such states, we allow the drift just at the boundary point to take an arbitrary value including zero.

Our contribution in this paper is three-fold:

1. The study of Mandjes et al. ${ }^{[14]}$ for the same multi-regime fluid queue excludes repulsive type boundaries and zero drift states. In this 
study, we relax these assumptions and allow repulsive and zero drift states in the analysis.

2. In the work of Mandjes et al. ${ }^{[14]}$, the differential equations and boundary conditions are derived for joint cumulative distribution functions $(\mathrm{CDF})$. In the current paper, we derive equivalent equations and conditions but this time in terms of joint probability density functions (PDF). We believe that this approach introduces an ease in notation and in computation.

3. In the spectral expansion approach of Tucker ${ }^{[2]}$ for the single regime fluid queue and of Mandjes et al. ${ }^{[14]}$ for the multi-regime case, the eigenvalues and eigenvectors of the systems of interest are first calculated and then a linear equation is formed using the boundary conditions so as to find the coefficients of the spectral expansion. This approach has two drawbacks; first, finding eigenvectors is generally known to be ill-conditioned especially when the corresponding eigenvalues are close. Moreover, the linear equation to solve for the coefficients of the spectral expansion also turns out to be ill-conditioned as demonstrated by Akar and Sohraby ${ }^{[8]}$ in the context of single-regime fluid queues. In this study, we propose to use the ordered Schur factorization as a numerical engine to alleviate the stability problems of the classical spectral expansion approach. We use this factorization to obtain a forward-backward decomposition of system behavior within each regime which eventually leads to a numerically stable scheme. The difference from Akar and Sohraby ${ }^{[8]}$ is that in this paper we use ordered Schur factorization as opposed to the matrix sign function as the numerical engine. While doing so, we can deal with zero drift states as well and numerical stability is attained even for the case of close to zero drift states.

The organization of the paper is as follows. We describe the multiregime feedback fluid queue in Section 2 and provide in compact form the boundary conditions for the steady state distribution of the underlying system. In Section 3, we describe the spectral expansion approach and study the number of unknowns and equations of the underlying system. Section 4 addresses the ordered Schur factorization approach for solving the system. We present various numerical examples to validate the effectiveness of the proposed approach in Section 5.

\section{STOCHASTIC MODEL}

We describe the general multi-regime feedback fluid queue model under consideration in this section. We let $C(t)$ denote the fluid level in the queue and $M(t)$ denote the state of the background process at time $t$. We identify thresholds $0=T^{(0)}<T^{(1)}<\cdots<T^{(K)}<\infty$ such that 
the buffer is said to be in regime $k$ if $T^{(k-1)}<C(t)<T^{(k)}$. The buffer is said to be at threshold $k$ when $C(t)=T^{(k)} \cdot T^{(0)}$ and $T^{(K)}$ are terminal boundary points whereas the other thresholds correspond to intermediate boundary points. We assume that the background process $\{M(t) ; t \geq 0\}$ has the state space $\{1,2, \ldots, M\}$. When the system is in regime $k$ (at threshold $\left.T^{(k)}\right)$ then the background process $M(t)$ behaves according to a Markov process with irreducible generator $Q^{(k)}\left(\widetilde{Q}^{(k)}\right)$. The drift while at state $m$, $1 \leq m \leq M$, in regime $k$ (at threshold $T^{(k)}$ ) is denoted by $r_{m}^{(k)}\left(\tilde{r}_{m}^{(k)}\right)$. We let $R^{(k)}\left(\widetilde{R}^{(k)}\right)$ to be the diagonal matrix of drifts in regime $k$ (at threshold $\left.T^{(k)}\right)$. The dynamics of the buffer content is given by:

$$
\frac{d C(t)}{d t}= \begin{cases}\max \left(0, \tilde{r}_{M(t)}^{(0)}\right) & \text { if } C(t)=0 \\ r_{M(t)} & \text { if } T^{(k-1)}<C(t)<T^{(k)}, 1 \leq k \leq K, \\ \tilde{r}_{M(t)} & \text { if } C(t)=T^{(k)}, 1 \leq k \leq K-1, \\ \min \left(0, \tilde{r}_{M(t)}^{(K)}\right) & \text { if } C(t)=T^{(K)} .\end{cases}
$$

Let $f^{(k)}(y, t)$ denote the row vector of transient joint probability density functions at time $t$ in regime $k$ for $1 \leq k \leq K$, i.e., $f^{(k)}(y, t)=$ $\left[f_{1}^{(k)}(y, t), f_{2}^{(k)}(y, t), \ldots, f_{M}^{(k)}(y, t)\right]$, where

$$
f_{m}^{(k)}(y, t)=\frac{\partial F_{m}^{(k)}(y, t)}{\partial y}, \quad T^{(k-1)}<y<T^{(k)}, \quad 1 \leq k \leq K, \quad 1 \leq m \leq M,
$$

and

$$
\begin{gathered}
F_{m}^{(k)}(y, t)=P(C(t) \leq y, M(t)=m), \quad T^{(k-1)}<y<T^{(k)} \\
1 \leq k \leq K, \quad 1 \leq m \leq M
\end{gathered}
$$

The steady-state joint density function can then be defined via taking the limit of (2) as $t \rightarrow \infty$, i.e., $f_{m}^{(k)}(y)=\lim _{t \rightarrow \infty} f_{m}^{(k)}(y, t)$. We then define the steady-state joint density vector

$$
f^{(k)}(y)=\left[\begin{array}{llll}
f_{1}^{(k)}(y) & f_{2}^{(k)}(y) & \cdots & f_{M}^{(k)}(y)
\end{array}\right] .
$$

Similarly, we define $F_{m}^{(k)}(y)=\lim _{t \rightarrow \infty} F_{m}^{(k)}(y, t)$ and $F^{(k)}(y)=\left[F_{1}^{(k)}(y)\right.$, $\left.F_{2}^{(k)}(y), \ldots, F_{M}^{(k)}(y)\right]$. Moreover, we define $c^{(k)}(t)$ to be the row vector of transient probability mass accumulations at the boundary point $T^{(k)}$ at time $t$ :

$$
c^{(k)}(t)=\left[\begin{array}{llll}
c_{1}^{(k)}(t) & c_{2}^{(k)}(t) \quad \cdots & c_{M}^{(k)}(t)
\end{array}\right], \quad 0 \leq k \leq K,
$$


where

$$
c_{m}^{(k)}(t)=P\left(C(t)=T^{(k)}, M(t)=m\right), \quad 0 \leq k \leq K, \quad 1 \leq m \leq M .
$$

The steady-state steady probability mass accumulations at the boundary points are defined by means of taking the limit of (6) as $t \rightarrow \infty$, i.e., $c_{m}^{(k)}=\lim _{t \rightarrow \infty} c_{m}^{(k)}(t), 0 \leq k \leq K, 1 \leq m \leq M$. We also define $c^{(k)}=$ $\left[c_{1}^{(k)}, c_{2}^{(k)}, \ldots, c_{M}^{(k)}\right]$. Finally, we define the joint CDF $F_{m}(y)$ for all $y$ such that

$$
F_{m}(y)=F_{m}^{(k)}(y), \quad 1 \leq m \leq M, \text { when } T^{(k-1)}<y<T^{(k)},
$$

and the buffer occupancy $\operatorname{CDF} F(y)=\left[F_{1}(y), F_{2}(y), \ldots, F_{M}(y)\right] e$ where $e$ is a column vector of ones of appropriate size. Note that by definition, $F_{m}(y)$ and $F(y)$ are right-continuous at the boundary points. The joint $\operatorname{PDF} f_{m}(y)$ and the buffer occupancy PDF $f(y)$ are defined accordingly. The steady solution to the feedback fluid queue involves the calculation of $f^{(k)}(\cdot)$ for $1 \leq k \leq K$ and $c^{(k)}$ for $0 \leq k \leq K$ which is the scope of the current paper. The feedback fluid queue of interest is illustrated in Figure 2.

Let $S_{+}^{(k)}, S_{0}^{(k)}$, and $S_{-}^{(k)}$ denote the set of states with positive, zero, and negative drifts, respectively, in regime $k$. Similarly, let $\widetilde{S}_{+}^{(k)}, \widetilde{S}_{0}^{(k)}$, and $\widetilde{S}_{-}^{(k)}$ denote the set of states with positive, zero, and negative drifts, respectively, at the boundary point $T^{(k)}$. Moreover, $S=S_{+}^{(k)} \cup S_{-}^{(k)} \cup S_{0}^{(k)}, \forall k$ denotes the set of all states. The intermediate boundary point $T^{(k)}$ is said to be a repulsive boundary point for state $m, 1 \leq m \leq M$, if $m \in S_{-}^{(k)} \cap S_{+}^{(k+1)}$. A state $m, 1 \leq m \leq M$, is a zero drift state in regime $k$ if $m \in S_{0}^{(k)}$. Finally, we define for $1 \leq k \leq K$ the mean drifts at regime $k$ :

$$
\mu^{(k)}=\pi^{(k)} R^{(k)} e
$$

where $\pi^{(k)}$ denotes the steady-state vector of $Q^{(k)}$, i.e.,

$$
\pi^{(k)} Q^{(k)}=0, \quad \pi^{(k)} e=1 .
$$

Given the notation above, we make the following assumptions on the drift configuration of the system by some of which we rule out anomalous situations.

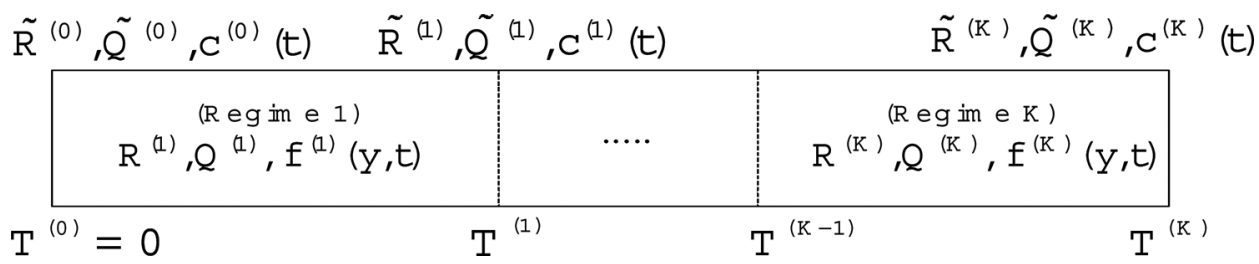

FIGURE 2 The first order multi-regime feedback fluid queue. 
A1) $S_{+}^{(k)} \neq \emptyset, S_{-}^{(k)} \neq \emptyset, 1 \leq k \leq K, \widetilde{S}_{+}^{(k)} \neq \emptyset, \widetilde{S}_{-}^{(k)} \neq \emptyset, 0 \leq k \leq K$.

A2) If $m \in S_{+}^{(k)} \cap S_{-}^{(k+1)}$ then $m \in \widetilde{S}_{0}^{(k)}, 1 \leq k \leq K-1$.

A3) The drifts at the boundary points for repulsive states are either zero, or left continuous, or right continuous.

A4) If $r_{m}^{(k)}=0$ then $\tilde{r}_{m}^{(k-1)}=\tilde{r}_{m}^{(k)}=0$.

A5) The drifts at other states are either left or right continuous at the corresponding boundary points.

A6) In all regimes, the mean drifts are nonzero, i.e., $\mu^{(k)} \neq 0,1 \leq k \leq K$.

Next, we justify the assumptions. Violation of A1 restricts the buffer content process to stay in a certain portion of the buffer or leads the content process to stick to a boundary point. It is also natural to select the drift at an intermediate boundary point $T^{(k)}$ as either left continuous $\left(\tilde{r}_{m}^{(k)}=r_{m}^{(k)}\right)$ or right continuous $\left(\tilde{r}_{m}^{(k)}=r_{m}^{(k+1)}\right)$ or simply zero $\left(\tilde{r}_{m}^{(k)}=0\right)$. For any $m$ and $k$ satisfying the condition $m \in S_{+}^{(k)} \cap S_{-}^{(k+1)}$, the buffer content would make infinitesimal oscillations around the boundary point $T^{(k)}$ at an infinite rate under the assumption of left and right continuities of drifts. We use the assumption A2 to avoid this situation. Similarly, A3 is used for boundary points with repulsive states. The assumption A4 removes the possibility of having more than one probability mass accumulation within arbitrarily small neighborhoods of boundary points. Additionally, we assume A5 for $m \in\left(S_{+}^{(k)} \cap S_{+}^{(k+1)}\right) \cup\left(S_{-}^{(k)} \cap S_{-}^{(k+1)}\right)$ in order to avoid a situation as the one described for A2. Finally, we avoid double eigenvalues at zero by ruling out zero mean drifts in A6. With these assumptions in place, we relax those of Ref. ${ }^{[14]}$ and allow zero drift states and boundary points that are repulsive for some states.

We next provide the main theorem of this paper that characterizes the solution to the steady-state joint density and the probability mass accumulations at the boundary points. We leave the proof of the theorem to Appendix A.

Theorem 2.1. The steady state joint density vector $f^{(k)}(\cdot)$ of the feedback Markov fluid queue satisfies the differential equations

$$
\frac{d}{d y} f^{(k)}(y) R^{(k)}=f^{(k)}(y) Q^{(k)}, \quad T^{(k-1)}<y<T^{(k)}, \quad 1 \leq k \leq K,
$$

along with the boundary conditions

$$
\begin{gathered}
c_{m}^{(0)}=0, \quad \forall m \in S_{+}^{(1)} \\
c_{m}^{(k)}=0, \quad \forall m \in\left(S_{+}^{(k)} \cap S_{+}^{(k+1)}\right) \cup\left(S_{-}^{(k)} \cap S_{-}^{(k+1)}\right), \quad 1 \leq k \leq K-1, \\
c_{m}^{(k)}=0, \quad \forall m \in\left(S_{-}^{(k)} \cap S_{+}^{(k+1)}\right) \cap\left(\widetilde{S}_{+}^{(k)} \cup \widetilde{S}_{-}^{(k)}\right), \quad 1 \leq k \leq K-1, \\
c_{m}^{(K)}=0, \quad \forall m \in S_{-}^{(K)},
\end{gathered}
$$




$$
\begin{gathered}
f^{(0)}(0+) R^{(1)}=c^{(0)} \widetilde{Q}^{(0)}, \\
f^{(k+1)}\left(T^{(k)}+\right) R^{(k+1)}-f^{(k)}\left(T^{(k)}-\right) R^{(k)}=c^{(k)} \widetilde{Q}^{(k)}, \quad 1 \leq k \leq K-1, \\
f_{m}^{(k)}\left(T^{(k)}-\right)=0, \quad \forall m \in S_{-}^{(k)} \cap\left(\widetilde{S}_{0}^{(k)} \cup \widetilde{S}_{+}^{(k)}\right), \quad 1 \leq k \leq K-1, \\
f_{m}^{(k+1)}\left(T^{(k)}+\right)=0, \quad \forall m \in\left(\widetilde{S}_{0}^{(k)} \cup \widetilde{S}_{-}^{(k)}\right) \cap S_{+}^{(k+1)}, \quad 1 \leq k \leq K-1, \\
f^{(K)}\left(T^{(K)}-\right) R^{(K)}=-c^{(K)} \widetilde{Q}^{(K)}, \\
\sum_{m=1}^{M}\left(\sum_{k=1}^{K} \int_{T^{(k-1)}+}^{T^{(k)}-} f_{m}^{(k)}(x) d x+\sum_{k=0}^{K} c_{m}^{(k)}\right)=1 .
\end{gathered}
$$

Remark 2.1. When compared with Mandjes et al. ${ }^{[14]}$ that uses cumulative distribution functions for the formulation of the boundary conditions, we use in this paper probability density functions which shorten the representation of these boundary conditions.

\section{SPECTRAL SOLUTION}

In this section, we present the spectral solution to feedback fluid queues. For this purpose, we note that in the special case of $S_{0}^{(k)}=\emptyset$, the general solution to (7) is given by:

$$
f^{(k)}(y)=\sum_{i \neq j} a_{i}^{(k)} \exp \left(\lambda_{i}^{(k)} y\right) \phi_{i}^{(k)}+a_{j}^{(k)} \phi_{j}^{(k)}, \quad T^{(k-1)}<y<T^{(k)}, \quad 1 \leq k \leq K
$$

where $\left(\lambda_{i}^{(k)}, \phi_{i}^{(k)}\right)$ is the $i$ th eigenvalue - left eigenvector pair of the matrix $Q^{(k)}\left(R^{(k)}\right)^{-1}$ and $\phi_{j}^{(k)}$ denotes the eigenvector corresponding to the zero eigenvalue. Here we assume simple eigenvalues as in Ref. ${ }^{[14]}$. In this solution, there are $M K$ unknown " $a$ " coefficients and $M K+M$ unknown " $c$ " coefficients (probability mass accumulations at the boundary points) that we need to find.

Remark 3.1. The general form of the solution when $S_{0}^{(k)} \neq \varnothing$ is the same as (18) whereas in this case we have $K M-\sum_{k}\left|S_{0}^{(k)}\right|$ unknown " $a$ " coefficients and $M K+M$ unknown " $c$ " coefficients, totalling $2 K M+M-$ $\sum_{k}\left|S_{0}^{(k)}\right|$ unknowns to be calculated.

Boundary conditions (12), (13), and (16) provide $M K+M$ equations. Now, we count the number of the other boundary conditions given in Theorem 2.1. The boundary conditions (8), (9), and (11) provide $\left|S_{+}^{(1)}\right|$, $\sum_{k=1}^{K-1}\left|\left(S_{+}^{(k)} \cap S_{+}^{(k+1)}\right) \cup\left(S_{-}^{(k)} \cap S_{-}^{(k+1)}\right)\right|$ and $\left|S_{-}^{(K)}\right|$ equations, respectively, whereas the boundary conditions (14) and (15) provide $\sum_{k=1}^{K-1} \mid S_{-}^{(k)} \cap$ 
$\left(\widetilde{S}_{0}^{(k)} \cup \widetilde{S}_{+}^{(k)}\right) \mid$ and $\sum_{k=1}^{K-1}\left|\left(\widetilde{S}_{0}^{(k)} \cup \widetilde{S}_{-}^{(k)}\right) \cap S_{+}^{(k+1)}\right|$ equations, respectively. Let $N_{r}$ denote the number of state-boundary pairs that possess a repulsive behavior. If the drifts in repulsive boundaries are selected as left continuous then condition (10) in Theorem 2.1 gives us $N_{r}$ boundary equations. In this case, we have

$$
\sum_{k=1}^{K-1}\left|S_{-}^{(k)} \cap\left(\widetilde{S}_{0}^{(k)} \cup \widetilde{S}_{+}^{(k)}\right)\right|=\sum_{k=1}^{K-1}\left|S_{-}^{(k)} \cap S_{0}^{(k+1)}\right|
$$

and

$$
\sum_{k=1}^{K-1}\left|\left(\widetilde{S}_{0}^{(k)} \cup \widetilde{S}_{-}^{(k)}\right) \cap S_{+}^{(k+1)}\right|=\sum_{k=1}^{K-1}\left|\left(S_{0}^{(k)} \cup S_{-}^{(k)}\right) \cap S_{+}^{(k+1)}\right|
$$

Therefore, the boundary conditions (8), (9), (11), (14), and (15) provide overall

$$
\begin{aligned}
\left|S_{+}^{(1)}\right| & +\left|S_{-}^{(K)}\right|+\sum_{k=1}^{K-1}\left|\left(S_{+}^{(k)} \cap S_{+}^{(k+1)}\right) \cup\left(S_{-}^{(k)} \cap S_{-}^{(k+1)}\right)\right|+\sum_{k=1}^{K-1}\left|S_{-}^{(k)} \cap S_{0}^{(k+1)}\right| \\
& +\sum_{k=1}^{K-1}\left|\left(S_{0}^{(k)} \cup S_{-}^{(k)}\right) \cap S_{+}^{(k+1)}\right| \\
= & \left|S_{+}^{(1)}\right|+\left|S_{-}^{(K)}\right|+\sum_{k=1}^{K-1}\left|S_{-}^{(k)} \cap\left(S_{0}^{(k+1)} \cup S_{-}^{(k+1)}\right)\right| \\
& +\sum_{k=1}^{K-1}\left|\left(S_{0}^{(k)} \cup S_{-}^{(k)} \cup S_{+}^{(k)}\right) \cap S_{+}^{(k+1)}\right| \\
= & \left|S_{+}^{(1)}\right|+\left|S_{-}^{(K)}\right|+\sum_{k=1}^{K-1}\left|S_{-}^{(k)}\right|-N_{r}+\sum_{k=1}^{K-1}\left|S_{+}^{(k+1)}\right| \\
= & \sum_{k=1}^{K}\left|S_{-}^{(k)}\right|+\sum_{k=1}^{K}\left|S_{+}^{(k)}\right|-N_{r}
\end{aligned}
$$

equations. One can similarly show the same number of equations when the drifts at repulsive states are right continuous. If the drifts at all repulsive boundaries are zero then in this case the boundary condition (10) does not provide any equations. In this case, we have however

$$
\sum_{k=1}^{K-1}\left|S_{-}^{(k)} \cap\left(\widetilde{S}_{0}^{(k)} \cup \widetilde{S}_{+}^{(k)}\right)\right|=\sum_{k=1}^{K-1}\left|S_{-}^{(k)} \cap\left(S_{0}^{(k+1)} \cup S_{+}^{(k+1)}\right)\right|,
$$


and

$$
\sum_{k=1}^{K-1}\left|\left(\widetilde{S}_{0}^{(k)} \cup \widetilde{S}_{-}^{(k)}\right) \cap S_{+}^{(k+1)}\right|=\sum_{k=1}^{K-1}\left|\left(S_{0}^{(k)} \cup S_{-}^{(k)}\right) \cap S_{+}^{(k+1)}\right|
$$

Therefore, the boundary conditions (8), (9), (11), (14), and (15) provide this time

$$
\begin{aligned}
\left|S_{+}^{(1)}\right| & +\left|S_{-}^{(K)}\right|+\sum_{k=1}^{K-1}\left|\left(S_{+}^{(k)} \cap S_{+}^{(k+1)}\right) \cup\left(S_{-}^{(k)} \cap S_{-}^{(k+1)}\right)\right| \\
& +\sum_{k=1}^{K-1}\left|S_{-}^{(k)} \cap\left(S_{0}^{(k+1)} \cup S_{+}^{(k+1)}\right)\right|+\sum_{k=1}^{K-1}\left|\left(S_{0}^{(k)} \cup S_{-}^{(k)}\right) \cap S_{+}^{(k+1)}\right| \\
= & \left|S_{+}^{(1)}\right|+\left|S_{-}^{(K)}\right|+\sum_{k=1}^{K-1}\left|S_{-}^{(k)} \cap\left(S_{0}^{(k+1)} \cup S_{-}^{(k+1)} \cup S_{+}^{(k+1)}\right)\right| \\
& +\sum_{k=1}^{K-1}\left|\left(S_{0}^{(k)} \cup S_{+}^{(k)} \cup S_{-}^{(k)}\right) \cap S_{+}^{(k+1)}\right| \\
= & \left|S_{+}^{(1)}\right|+\left|S_{-}^{(K)}\right|+\sum_{k=1}^{K-1}\left|S_{-}^{(k)}\right|+\sum_{k=1}^{K-1}\left|S_{+}^{(k+1)}\right| \\
= & \sum_{k=1}^{K}\left|S_{-}^{(k)}\right|+\sum_{k=1}^{K}\left|S_{+}^{(k)}\right|
\end{aligned}
$$

equations. Finally, in either case (left continuous or right continuous or zero drift at repulsive states), Theorem 2.1 provides overall $2 K M-$ $\sum_{k}\left|S_{0}^{(k)}\right|+1$ boundary conditions together with the normalization condition (17). One of these equations is redundant as in Mandjes et al. ${ }^{[14]}$ and Akar and Sohraby ${ }^{[8]}$ and the number of equations and the number of unknowns are identical making it possible to uniquely solve the system.

\section{NUMERICAL ALGORITHM}

The spectral approach of the previous section has a number of drawbacks:

i) The approach fails when the eigenvalues are not simple,

ii) Finding the eigenvectors of a general non-symmetric matrix is known to be ill-conditioned especially for eigenvalues close to each other, 
iii) The linear equation to solve the unknowns of the spectral expansion in (18) is known to be ill-conditioned; see the numerical experimentation of Akar and Sohraby ${ }^{[8]}$.

Based on these observations, we use in this paper the so-called ordered Schur form as opposed to the Jordan form, the latter required for finding all eigenvectors. We refer the reader to Appendix A2.1 for a brief summary of the ordered Schur form.

Throughout this section, we will focus on a particular regime $k$. In Theorem 2.1, we have shown that the probability density function satisfies for regime $k$ the following differential equation:

$$
\frac{d}{d y} f^{(k)}(y) R^{(k)}=f^{(k)}(y) Q^{(k)}, \quad T^{(k-1)}<y<T^{(k)} .
$$

Assume that $\left|S_{0}^{(k)}\right|=0$. Then under the irreducible background process and $\mu^{(k)} \neq 0$ assumptions, we have one eigenvalue at the origin, $\left|S_{+}^{(k)}\right|-1$ eigenvalues with negative real parts and $\left|S_{-}^{(k)}\right|$ eigenvalues with positive real parts in case $\mu^{(k)}<0$. When $\mu^{(k)}>0$, we again have one eigenvalue at the origin but $\left|S_{+}^{(k)}\right|$ eigenvalues with negative real parts and $\left|S_{-}^{(k)}\right|-1$ eigenvalues with positive real parts. From continuity arguments, when $\mu^{(k)}=0$, we have two eigenvalues at the origin and $\left|S_{+}^{(k)}\right|-1$ eigenvalues with negative real parts and $\left|S_{-}^{(k)}\right|-1$ eigenvalues with positive real parts. These arguments also hold for the case $\left|S_{0}^{(k)}\right| \neq 0$ but in terms of the number of generalized eigenvalues since $R^{(k)}$ in (19) is not invertible; see Akar and Sohraby ${ }^{[8]}$ for a description of generalized eigenvalues and eigenvectors. As stated before, we assume in this paper that $\mu^{(k)} \neq 0$ to avoid double eigenvalues at the origin. Let us also assume without loss of generality that the states are ordered in such a way in regime $k$ that states with zero drifts are numbered after the ones with nonzero drifts. If not, one can employ a similarity transformation to do so. Under this assumption, (7) can then be rewritten as:

$$
\begin{gathered}
\frac{d f_{n}^{(k)}(y)}{d y} R_{n}^{(k)}=f_{n}^{(k)}(y) \underbrace{\left(Q_{n n}^{(k)}-Q_{n z}^{(k)}\left(Q_{z z}^{(k)}\right)^{-1} Q_{z n}^{(k)}\right)}_{Q_{n}^{(k)}}, \\
f_{z}^{(k)}(y)=-f_{n}^{(k)}(y) Q_{n z}^{(k)}\left(Q_{z z}^{(k)}\right)^{-1},
\end{gathered}
$$

where we have

$$
f^{(k)}(y)=\left[f_{n}^{(k)}(y) \quad f_{z}^{(k)}(y)\right],
$$


and

$$
R^{(k)}=\left[\begin{array}{cc}
R_{n}^{(k)} & 0 \\
0 & 0
\end{array}\right], \quad Q^{(k)}=\left[\begin{array}{cc}
Q_{n n}^{(k)} & Q_{n z}^{(k)} \\
Q_{z n}^{(k)} & Q_{z z}^{(k)}
\end{array}\right]
$$

Here $f_{n}^{(k)}$ and $f_{z}^{(k)}$ refer to the steady state joint probability density vectors corresponding to $n^{(k)}=\left|S_{+}^{(k)}\right|+\left|S_{-}^{(k)}\right|$ states with nonzero drifts and $z^{(k)}=$ $\left|S_{0}^{(k)}\right|$ states with zero drifts, respectively. The differential equation (20) can then be rewritten as

$$
\frac{d}{d y} f_{n}^{(k)}(y)=f_{n}^{(k)}(y) Q_{n}^{(k)}\left(R_{n}^{(k)}\right)^{-1}=f_{n}^{(k)}(y) A^{(k)}
$$

Using the procedure described in Appendix A2.1, one can find a matrix $Y^{(k)}$ such that

$$
\left(Y^{(k)}\right)^{-1} A^{(k)} Y^{(k)}=\left[\begin{array}{lll}
0 & & \\
& A_{-}^{(k)} & \\
& & A_{+}^{(k)}
\end{array}\right]
$$

where the real parts of the eigenvalues of $A_{-}^{(k)}$ and $A_{+}^{(k)}$ are negative and positive, respectively. This decomposition leads us to

$$
\frac{d}{d y} f_{n}^{(k)}(y) Y^{(k)}=f_{n}^{(k)}(y) Y^{(k)}\left(Y^{(k)}\right)^{-1} A^{(k)} Y^{(k)} .
$$

We then define

$$
z^{(k)}(y)=f_{n}^{(k)}(y) Y^{(k)}
$$

where

$$
z^{(k)}(y)=\left[\begin{array}{lll}
z_{0}^{(k)}(y) & z_{-}^{(k)}(y) & z_{+}^{(k)}(y)
\end{array}\right]
$$

with appropriate sizes for $z_{0}^{(k)}(y), z_{-}^{(k)}(y)$ and $z_{+}^{(k)}(y)$. We then obtain the following solutions

$$
\begin{gathered}
z_{0}^{(k)}(y)=a_{0}^{(k)} \\
z_{-}^{(k)}(y)=z_{-}^{(k)}\left(T^{(k-1)}\right) \exp \left\{A_{-}^{(k)}\left(y-T^{(k-1)}\right)\right\} \\
z_{+}^{(k)}(y)=z_{+}^{(k)}\left(T^{(k)}\right) \exp \left\{-A_{+}^{(k)}\left(T^{(k)}-y\right)\right\}
\end{gathered}
$$


where $a_{0}^{(k)}$ is a constant. A suitable decomposition of matrix $\left(Y^{(k)}\right)^{-1}$ is

$$
\left(Y^{(k)}\right)^{-1}=\left[\begin{array}{c}
\tilde{Y}_{0}^{(k)} \\
\widetilde{Y}_{-}^{(k)} \\
\tilde{Y}_{+}^{(k)}
\end{array}\right],
$$

and the relation $f_{n}^{(k)}(y)=z^{(k)}(y)\left(Y^{(k)}\right)^{-1}$ yields

$$
\begin{aligned}
f_{n}^{(k)}(y)= & a_{0}^{(k)} \tilde{Y}_{0}^{(k)}+z_{-}^{(k)}\left(T^{(k-1)}\right) \exp \left\{A_{-}^{(k)}\left(y-T^{(k-1)}\right)\right\} \tilde{Y}_{-}^{(k)} \\
& +z_{+}^{(k)}\left(T^{(k)}\right) \exp \left\{-A_{+}^{(k)}\left(T^{(k)}-y\right)\right\} \widetilde{Y}_{+}^{(k)}
\end{aligned}
$$

Using the identities (21) and (22), we finally write

$$
\begin{aligned}
f^{(k)}(y)= & a_{0}^{(k)} L_{0}^{(k)}+a_{-}^{(k)} \exp \left\{A_{-}^{(k)}\left(y-T^{(k-1)}\right)\right\} L_{-}^{(k)} \\
& +a_{+}^{(k)} \exp \left\{-A_{+}^{(k)}\left(T^{(k)}-y\right)\right\} L_{+}^{(k)}
\end{aligned}
$$

where

$$
\begin{aligned}
& a_{-}^{(k)}=z_{-}^{(k)}\left(T^{(k-1)}\right), \\
& a_{+}^{(k)}=z_{+}^{(k)}\left(T^{(k)}\right), \\
& L_{i}^{(k)}=\left[\tilde{Y}_{i}^{(k)},-\widetilde{Y}_{i}^{(k)} Q_{n z}^{(k)}\left(Q_{z}^{(k)}\right)^{-1}\right], \quad i=0,-,+.
\end{aligned}
$$

The unknown coefficients of the expression (28) are $a_{0}^{(k)}, a_{-}^{(k)}$, and $a_{+}^{(k)}$. In order to calculate these coefficients, we suggest to feed the expression for $f^{(k)}(y)$ in (28) into the boundary conditions of Theorem 2.1 as opposed to the spectral expansion given in (18). With this approach, we not only eliminate the numerical stability problems arising in the process of finding eigenvectors but also the linear equation to solve the coefficients in the expression (28) becomes stable since all the involved matrices, e.g., $A_{-}^{(k)}$ and $-A_{+}^{(k)}$, are stability matrices, i.e., their eigenvalues have negative real parts.

Remark 4.1. The extension of Theorem 2.1 to the infinite buffer case is straightforward. If the length of regime $K$ is infinite, i.e., $T^{(K)}=\infty$, and if the stability condition

$$
\pi^{(K)} R^{(K)} e<0
$$

holds, then with appropriate normalization condition instead of the boundary conditions (11) and (16), we have to use the boundary 
condition:

$$
a_{i}^{(K)}=0, \quad \forall i: \Re\left\{\lambda_{i}\right\}>0
$$

for the spectral solution described in the previous section or equivalently

$$
a_{+}^{(K)}=0
$$

for the numerical algorithm we propose.

\section{NUMERICAL EXAMPLES}

In the following sections, we present an example (Example 1) for systems including zero drift states and states with repulsive type boundaries; another example (Example 2) for the comparison of stability of the conventional spectral approach and ordered Schur factorization approaches; an example (Example 3) showing that the zero mean drift case can be obtained as the limiting solution of nonzero mean drifts; and finally an example (Example 4) demonstrating the scalability of our proposed approach to large state-spaces. We use MATLAB 7.0 for implementing the numerical algorithm and the Matlab programs are available in Kankaya and Akar ${ }^{[17]}$ for public use.

\subsection{Example 1}

We consider the following five state example with a finite buffer of size two. The buffer consists of two regimes 1 and 2, corresponding to the intervals $(0,1)$ and $(1,2)$, respectively. We let

$$
R^{(1)}=\operatorname{diag}(1,-2,2,-4,0), \quad R^{(2)}=\operatorname{diag}(-1,1,3,-1,2),
$$

and

$$
\widetilde{Q}^{(0)}=Q^{(1)}=\widetilde{Q}^{(1)}=Q^{(2)}=\widetilde{Q}^{(2)}=\left[\begin{array}{ccccc}
-4 & 1 & 1 & 1 & 1 \\
1 & -4 & 1 & 1 & 1 \\
1 & 1 & -4 & 1 & 1 \\
1 & 1 & 1 & -4 & 1 \\
1 & 1 & 1 & 1 & -4
\end{array}\right]
$$

In this example, the first state has an absorbing boundary at the boundary point 1 ; the second state has a repulsive type boundary behavior at the same boundary point which is assumed to have zero drift in this state. The drifts in the third and the fourth states do not change their signs across the intermediate boundary point and therefore they are emitting. 
The fifth state is a zero drift state in regime 1. Using the assumptions, the drifts at the boundary point 1 in the first and the fifth states should be zero. The drifts at the same boundary point in the third and the fourth states can be either left or right continuous, which do not change the results. Finally, the drift at the same point in the second state can be selected as left or right continuous, or zero, which affects the results. However, we let this drift to be equal to zero for this example. The functions $F_{m}(y)$ are plotted for $m=1, \ldots, 5$ with respect to the buffer occupancy $y$ in Figure 3. We observe that the analytical results agree with the simulation results confirming the boundary conditions given in Theorem 2.1 for various types of states as stated in Theorem 2.1. Note that the derivative of $F_{2}(y)$ is zero on both sides of the boundary point 1 . Furthermore, the derivative of $F_{5}(y)$ on the right side of boundary point 1 approaches to zero.

\subsection{Example 2}

In this example, we compare the stability of the conventional spectral expansion approach and the ordered Schur factorization approach while the drift in a certain state for a given regime approaches to zero. For this purpose, we construct a simple three-state two-regime example for a

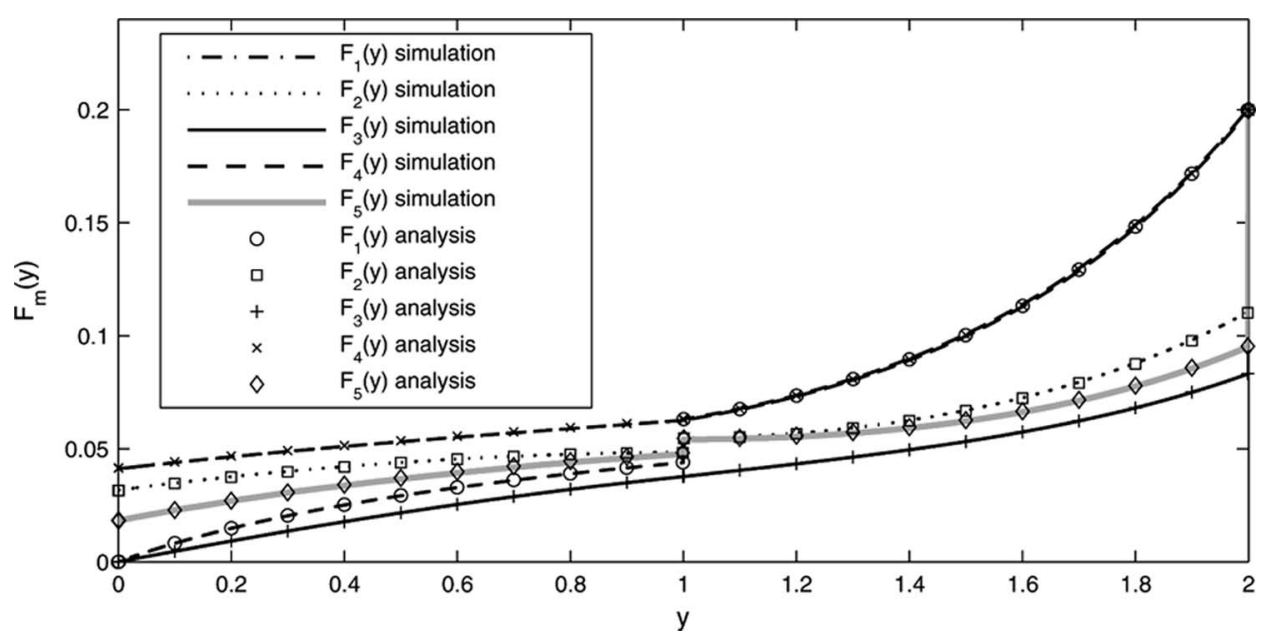

FIGURE 3 The quantity $F_{m}(y)$ plotted for $m=1, \ldots, 5$ as a function of the buffer occupancy $y$ in Example 1. 
buffer of size three consisting of two regimes 1 and 2 , corresponding to the intervals $(0,1)$ and $(1,3)$, respectively. We let

$$
\begin{aligned}
& \widetilde{Q}^{(0)}=Q^{(1)}=\left[\begin{array}{ccc}
-2 & 1 & 1 \\
1 & -2 & 1 \\
1 & 1 & -2
\end{array}\right], \quad \widetilde{Q}^{(1)}=Q^{(2)}=\widetilde{Q}^{(2)}=\left[\begin{array}{ccc}
-4 & 2 & 2 \\
2 & -4 & 2 \\
2 & 2 & -4
\end{array}\right], \\
& R^{(1)}=\operatorname{diag}(r,-1,2), \quad R^{(2)}=\operatorname{diag}(-0.5,-1,1),
\end{aligned}
$$

and

$$
\widetilde{R}^{(0)}=\operatorname{diag}(0,0,2), \quad \widetilde{R}^{(1)}=\operatorname{diag}(r,-1,2), \quad \widetilde{R}^{(2)}=\operatorname{diag}(-0.5,-1,0),
$$

where the variable $r<0$.

In Figure 4, the CDF of the buffer occupancy $F(y)$ is obtained using the ordered Schur factorization and is compared against simulations for three different values of $r=-0.05,-0.005,0$. We show in this figure that we get accurate results even for very small values of the drift parameter $r$. Furthermore, Figure 4 demonstrates that the solution of the fluid queue with $r<0$ as $r \rightarrow 0$ converges to the solution of the system for $r=0$ that is solved by using the boundary conditions given in Theorem 2.1.

In Figure 5, the buffer occupancy PDF is obtained using the ordered Schur factorization, together with the spectral approach and simulations for the case $r=-0.05$. It is clear from Figure 5 that the spectral approach does not provide accurate results in this case whereas our proposed method continues to give correct results even for very small absolute values of $r$.

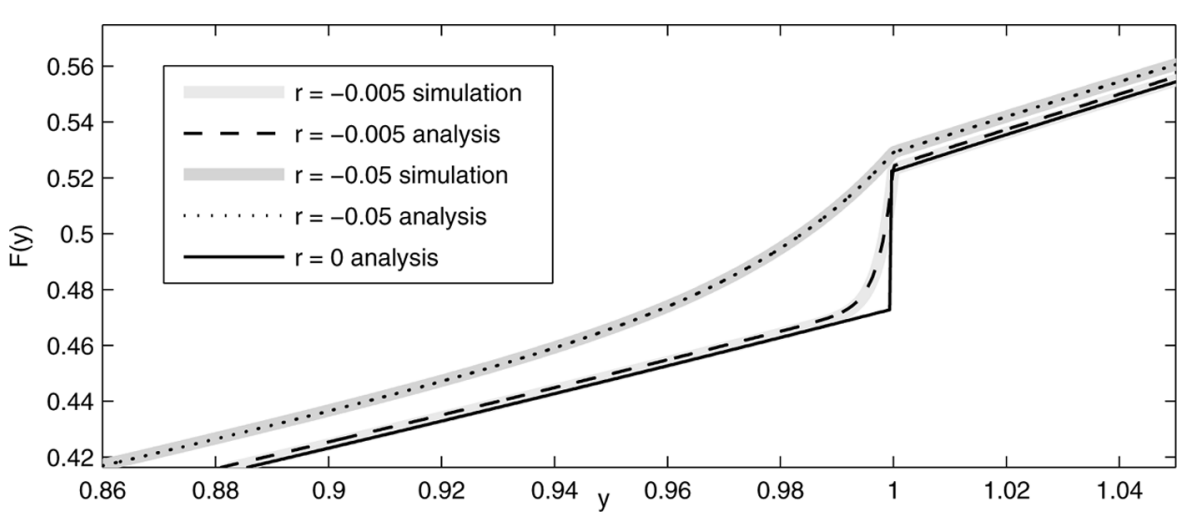

FIGURE 4 The quantity $F(y)$ plotted as a function of the buffer occupancy $y$ in the vicinity of the boundary point $y=1$ in Example 2 . 


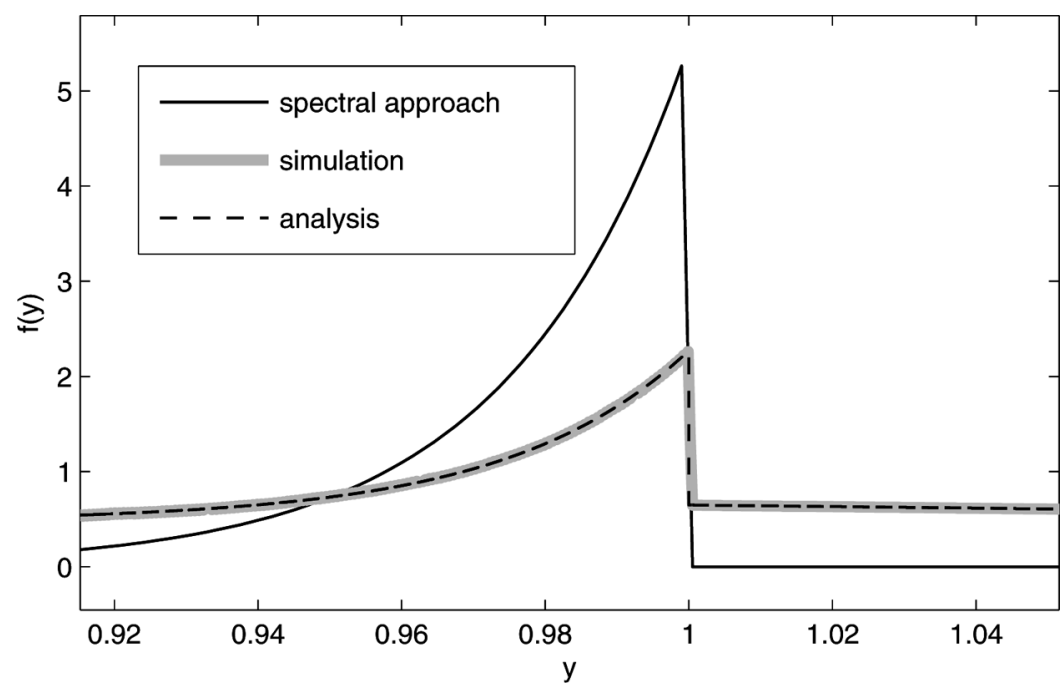

FIGURE 5 Comparison of the spectral approach and the ordered Schur factorization methods for $r=-0.05$ in Example 2.

\subsection{Example 3}

In this example, we vary the $r$ parameter of the previous example in the neighborhood of $r=-1$ at which the mean drift in the first regime is exactly zero. In Figure 6, we plot $F(y)$ for three values of $r$, namely for $r=$ $-0.8, r=-0.9$ and $r=-0.99$, so as to compare with the simulation result for $r=-1$. Figure 6 clearly demonstrates that the analysis results converge

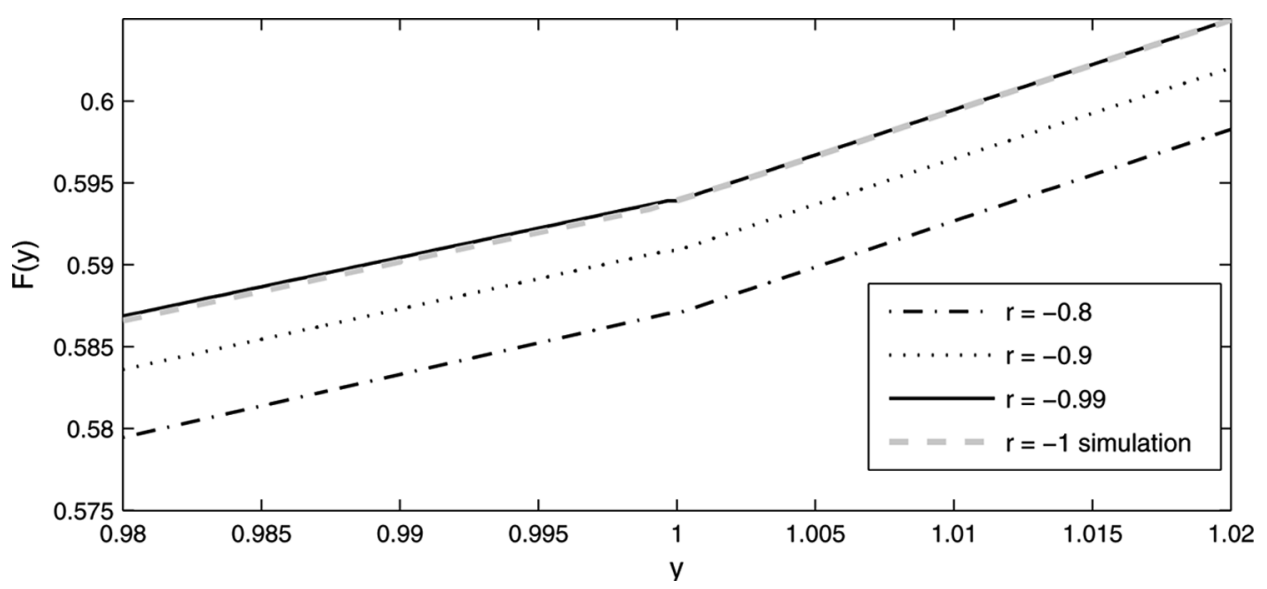

FIGURE 6 The quantity $F(y)$ plotted as a function of the buffer occupancy $y$ in the vicinity of the boundary point $y=1$ in Example 3 . 
to the simulation result as $r \rightarrow-1$ without having any computational difficulty in the vicinity of a zero mean drift regime.

\subsection{Example 4}

In this example, we consider a telecommunications link applying an adaptive admission control policy to the link buffer with capacity $c$ and buffer size $B$. We assume there are $N$ registered users. A user is modeled as an ON-OFF source which generates a workload at rate $r$ when the source is $\mathrm{ON}$. When the source becomes ON, the link buffer is to make a decision on whether to accept this workload as a whole to the link buffer. In the OFF state, the source does not generate any workload and no decisions are made. The alternating $\mathrm{ON}$ times and the OFF times are exponentially distributed with means $1 / \mu$ and $1 / \lambda$, respectively, for each source. The workload admission policy uses the buffer occupancy as the admission criterion; all new workloads are accepted when the buffer occupancy is below a predetermined threshold $T$ and rejected otherwise. In this way, the adaptive system prevents random losses due to buffer overflow. It is clear that this problem fits into a two-regime feedback fluid queueing system. The modulating process is governed by the number of admitted users. The state transition diagrams of this Markov chain are illustrated in Figure 7 depending on whether the buffer occupancy is below or above $T$. An aggressive admission policy, i.e., $T \rightarrow B$ reduces workload blocking probability but introduces buffer overflow which leads to random losses among all admitted workloads. On the other hand, for a conservative policy, i.e., $T \rightarrow 0$, buffer overflow probabilities will be reduced at the expense of increased workload blocking probabilities. In order to quantify this tradeoff, we define $T_{\max }$ to be the maximum value of the threshold parameter $T$ below which the buffer overflow probability is kept below

(a)

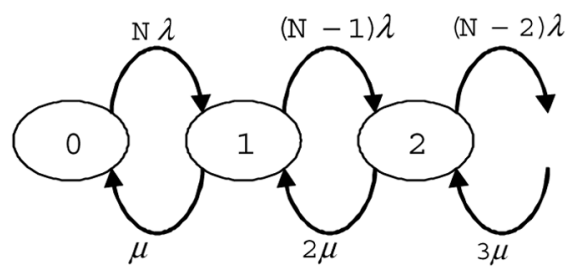

(b)

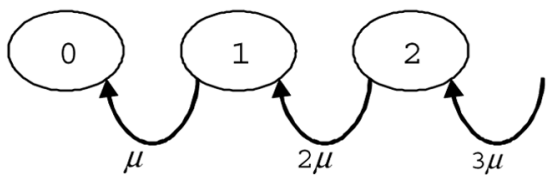

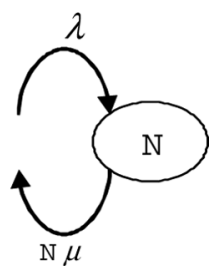

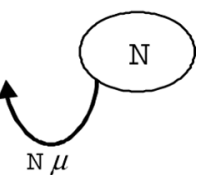

FIGURE 7 State transition diagram for Example 4 when the buffer occupancy is a) below $T$ b) above $T$. 


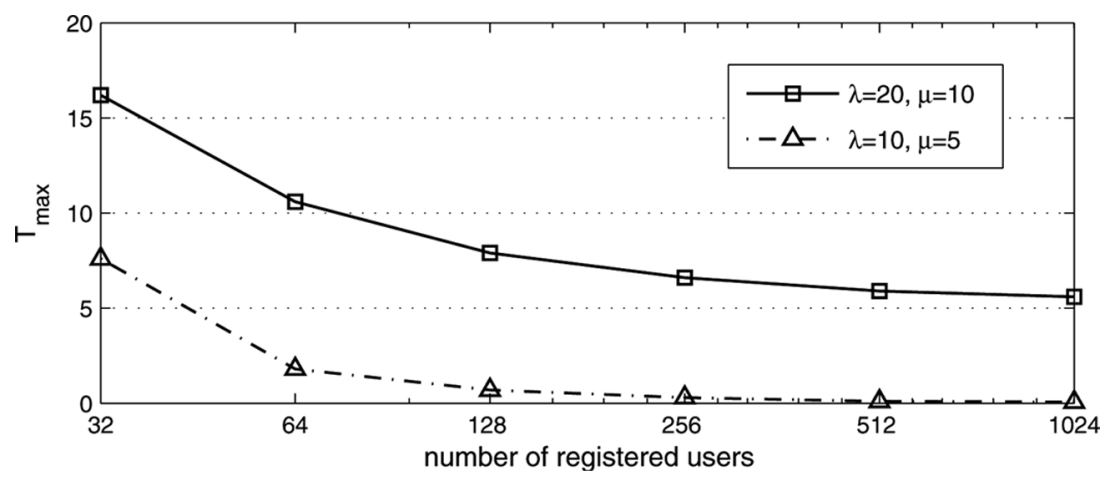

FIGURE 8 The $T_{\max }$ value in ms. for Example 4.

an acceptable value say $10^{-3}$. As an example, we fix $r=32 \mathrm{~Kb} / \mathrm{sec}, c=$ $500 \mathrm{~Kb} / \mathrm{sec}$, and $B=50 \mathrm{~ms}$. and we plot $T_{\max }$ (in ms.) as a function of the number of registered users in Figure 8 for two scenarios i) $\lambda=20$, $\mu=10$ ii) $\lambda=10, \mu=5$, the first scenario being for a more dynamic scenario where the transition rates are relatively higher. It is clear that one can employ a more aggressive admission policy with fewer registered users and more dynamic environment. We also note that we can solve two-regime feedback fluid queues with $2^{10}$ states (see Figure 8) without encountering any numerical problems.

Remark 5.4.1. The infinitesimal generator in the second regime has an absorbing state with all other states being transient. Since the number of absorbing states is one, the eigenvalue distribution, i.e., the number of eigenvalues at the origin, left half plane, and right half plane, remains intact and therefore the spectral approach and the Schur factorization approach described in this paper can be used with no modifications for this example.

\section{CONCLUSIONS}

In this paper, we study multi-regime Markov fluid queues for which we derive the boundary conditions in terms of joint densities. We use the ordered Schur factorization as the numerical engine to find the steadystate distribution of the system. The proposed method is numerically stable and we do not encounter stability problems for scenarios that are problematic for the traditional spectral approach. Moreover, problems with large state spaces are within reach using the proposed approach. For example, we have been able to obtain accurate results for a two-regime feedback fluid queue with $2^{10}$ states without facing any computational difficulty. 


\section{A1. APPENDIX A}

\section{A1.1. Proof of Theorem 2.1}

The differential equation (7) and the boundary conditions (8) through (13) and the two conditions (16) and (17) can be proven by using the results of Mandjes et al. ${ }^{[14]}$; the remaining two conditions (14) and (15) are related to boundary points with repulsive states and regimes with zero drift states. Mandjes et al. ${ }^{[14]}$ find the following ordinary differential equations of the joint distribution functions for multi-regime feedback fluid queues:

$$
\begin{aligned}
\frac{d}{d y} F^{(k)}(y) R^{(k)}= & \left(F^{(k)}(y)-F^{(k)}\left(T^{(k-1)}\right)\right) Q^{(k)} \\
& +\left(F^{(k)}\left(T^{(k-1)}\right)-F^{(k-1)}\left(T^{(k-1)}-\right)\right) \widetilde{Q}^{(k-1)} \\
& +\left(F^{(k-1)}\left(T^{(k-1)}-\right)-F^{(k-1)}\left(T^{(k-2)}\right)\right) Q^{(k-1)} \\
& \vdots \\
& +\left(F^{(1)}\left(T^{(1)}-\right)-F^{(1)}\left(T^{(0)}\right)\right) Q^{(1)}+F^{(1)}\left(T^{(0)}\right) \widetilde{Q}^{(0)}, \\
& T^{(k-1)}<y<T^{(k)}, \quad 1 \leq k \leq K .
\end{aligned}
$$

Equation (7) can be obtained by just differentiating both sides of (A.31) with respect to $y$. The boundary conditions (8)-(11) are immediate consequences of the fact that if nothing forces the content to stay at a point then the probability mass accumulation will be zero at that point. These conditions are equivalent to the continuity conditions in the cumulative distribution function formulation of Mandjes et al. ${ }^{[14]}$. Equation (12) can be obtained by just writing (A.31) for the first regime and equating the variable $y$ to $T^{(0)}+$ in that equation. Equation (13) can be obtained by writing (A.31) for regime $k$ and equating the variable $y$ to $T^{(k)}$ - in this equation and then writing (A.31) for regime $k+1$ and equating the variable $y$ to $T^{(k)}+$, and then subtracting the former one from the latter. Equation (16) can be obtained by writing (A.31) for the $K$ th regime and equating the variable $y$ to $T^{(K)}-$ in this equation and then subtracting it side by side from the following equation obtained by Mandjes et al. ${ }^{[14]}$ :

$$
\begin{aligned}
0= & \pi \widetilde{Q}^{(K)}+F^{(K)}\left(T^{(K)}-\right)\left(Q^{(K)}-\widetilde{Q}^{(K)}\right) \\
& +F^{(K)}\left(T^{(K-1)}\right)\left(\widetilde{Q}^{(K-1)}-Q^{(K)}\right) \\
& +F^{(K-1)}\left(T^{(K-1)}-\right)\left(Q^{(K-1)}-\widetilde{Q}^{(K-1)}\right) \\
& \vdots \\
& +F^{(1)}\left(T^{(1)}-\right)\left(Q^{(1)}-\widetilde{Q}^{(1)}\right)+F^{(1)}\left(T^{(0)}\right)\left(\widetilde{Q}^{(0)}-Q^{(1)}\right),
\end{aligned}
$$


where $\pi$ is the vector of steady state probabilities at the uppermost boundary point of the queue. The Equation (17) is the normalization condition, i.e., the sum of all probabilities accumulated at the boundary points and integral of the probability densities over all regimes is equal to 1 .

Next, we derive the boundary condition (14). For this purpose, we write the forward Kolmogorov equations for the following function defined for $T^{(k-1)}<y<T^{(k)}$ just below the boundary point $T^{(k)}$ :

$$
\overleftarrow{P}_{m}^{(k)}(x, y, t)=P(x \leq C(t)<y, M(t)=m), \quad m \in S_{-}^{(k)} \cap\left(\widetilde{S}_{0}^{(k)} \cup \widetilde{S}_{+}^{(k)}\right) .
$$

We first write the left hand side of the Kolmogorov equation:

$$
\begin{aligned}
& \overleftarrow{P}_{m}^{(k)}\left(T^{(k)}+r_{m}^{(k)} \Delta t, T^{(k)}, t+\Delta t\right) \\
& \quad=P\left(T^{(k)}+r_{m}^{(k)} \Delta t \leq C(t+\Delta t)<T^{(k)}, M(t+\Delta t)=m\right)
\end{aligned}
$$

We assume that $\Delta t$ is sufficiently small and the probability of more than one transition in the background process within time interval $[t, t+\Delta t]$ is $o(\Delta t)$. Since $m \in S_{-}^{(k)} \cap\left(\widetilde{S}_{0}^{(k)} \cup \widetilde{S}_{+}^{(k)}\right)$, according to the assumptions made in Section 2, $r_{m}^{(k+1)}$ is either zero or positive. For both of these cases, $C(t+\Delta t)$ cannot be in the interval $\left[T^{(k)}+r_{m}^{(k)} \Delta t, T^{(k)}\right)$ without any state transitions in the background process in the time interval $[t, t+\Delta t]$. For the case of one transition in the background process, the interval of the content process at time $t$ does not constitute any probability mass accumulation. The corresponding transition from any other state $n$ can occur only in regime $k$ with probability $O(\Delta t)$. Therefore, if we divide both sides of the forward Kolmogorov equation by $\Delta t$ and take the limit of both sides as $\Delta t \rightarrow 0$ we obtain zero on the right hand side. However, the left hand side becomes

$$
\begin{aligned}
& \lim _{\Delta t \rightarrow 0} \frac{\overleftarrow{P}_{m}^{(k)}\left(T^{(k)}+r_{m}^{(k)} \Delta t, T^{(k)}, t+\Delta t\right)}{\Delta t}=-r_{m}^{(k)} f_{m}^{(k)}\left(T^{(k)}-, t\right), \\
& \quad m \in S_{-}^{(k)} \cap\left(\widetilde{S}_{0}^{(k)} \cup \widetilde{S}_{+}^{(k)}\right) .
\end{aligned}
$$

Therefore by equating (A.35) to zero, (14) can be obtained. Derivation of (15) is quite similar to the derivation of (14). For this case we need to define for $T^{(k)}<y<T^{(k+1)}$ the following function:

$$
\vec{P}_{m}^{(k+1)}(x, y, t)=P(x<C(t) \leq y, M(t)=m), \quad m \in\left(\widetilde{S}_{0}^{(k)} \cup \widetilde{S}_{-}^{(k)}\right) \cap S_{+}^{(k+1)},
$$

and write the forward Kolmogorov equations for $x=T^{(k)}$ and $y=T^{(k)}+$ $r_{m}^{(k)} \Delta t$. 


\section{A2. APPENDIX B}

\section{A2.1. Ordered Schur Form}

We start by stating without proof the following theorem based on Golub and Van Loan ${ }^{[18]}$ (Ch. 7).

Theorem A2.1.1. Let $A$ be a real square matrix. Then, there exists an orthogonal matrix $Z$ such that

$$
Z^{T} A Z=D=\left[\begin{array}{cc}
D_{11} & D_{12} \\
0 & D_{22}
\end{array}\right],
$$

where $D$ is upper block triangular with either 1-by-1 or 2-by-2 diagonal blocks (hence the term quasi-triangular), respectively, comprising real and complex conjugate eigenvalues of $A$ in any desired order. The matrix $D$ is said to be in ordered real Schur form, and the columns of matrix $Z$ are referred to as Schur vectors.

Remark A2.1.1. In the ordered Schur form, any ordering policy can be used; for example eigenvalues with negative real parts can be placed at the upper-left corner $\left(D_{11}\right.$ has all its eigenvalues in the left half plane) and those with positive real parts at the lower-right corner $\left(D_{22}\right.$ has all its eigenvalues in the right half plane).

Given the matrix $A$ and its ordered Schur form as in (B.37), suppose that we want to find a nonsingular $Y$ such that

$$
Y^{-1} A Y=\left[\begin{array}{cc}
D_{11} & 0 \\
0 & D_{22}
\end{array}\right]
$$

by eliminating the upper-right partition $D_{12}$ of matrix $D$. To find $Y$, we block diagonalize the matrix $D$ of (B.37) as follows:

$$
\left[\begin{array}{cc}
I & X \\
0 & I
\end{array}\right]\left[\begin{array}{cc}
D_{11} & D_{12} \\
0 & D_{22}
\end{array}\right]\left[\begin{array}{cc}
I & -X \\
0 & I
\end{array}\right]=\left[\begin{array}{cc}
D_{11} & 0 \\
0 & D_{22}
\end{array}\right],
$$

where $X$ should be found by solving the Sylvester matrix equation (Golub and Van Loan ${ }^{[18]}$, Ch. 7)

$$
D_{11} X-X D_{22}=D_{12},
$$

for quasi-triangular $D_{11}$ and $D_{22}$. The existence of a unique solution $X$ stems from the spectrum disjointness of $D_{11}$ and $D_{22}$. Then, $Y$ follows from 
(B.37), (B.38), and (B.39) as

$$
Y=\left[\begin{array}{ll}
Z_{11} & Z_{12} \\
Z_{21} & Z_{22}
\end{array}\right]\left[\begin{array}{cc}
I & -X \\
0 & I
\end{array}\right]=\left[\begin{array}{cc}
Z_{11} & Z_{12}-Z_{11} X \\
Z_{21} & Z_{22}-Z_{21} X
\end{array}\right],
$$

where $Z$ is partitioned accordingly. For more information on ordered real Schur factorization and block diagonalization, we refer the reader to ${ }^{[18]}$ (Ch. 7).

We also note that appropriate computational routines are readily available in LAPACK $^{[19]}$ and MATLAB ${ }^{[20]}$ both for ordered real Schur factorization and for solving the Sylvester matrix equation (B.40) with upper quasi-triangular coefficient matrices $D_{11}$ and $D_{22}$. We note that obtaining the real Schur form is known to be backward stable and has a complexity of $a n^{3}$, where $a$ accounts for the iteration in the algorithm and may vary between 10 and 25; see Van Dooren ${ }^{[21]}$. On the other hand, for the computation of the Jordan form that requires calculation of all eigenvectors, there are no results of guaranteed backward stability and the complexity of this decomposition is much higher than that of the Schur decomposition; see Van Dooren ${ }^{[21]}$. For this reason, Van Dooren ${ }^{[21]}$ strongly recommends not to use the Jordan decomposition whenever one can use the more reliable Schur form as we do in the current paper. We note that this view is also shared by Bai et al. ${ }^{[22]}$ in which the standard serial algorithm for the eigen decomposition problem is the ordered Schur factorization due to its well-established numerical stability. We also note the alternative matrix sign function method ${ }^{[22]}$ which is not as numerically stable for the case of close to zero drifts.

Assume that the square matrix $A$ has eigenvalues at the origin. It is also clear that one can use the ordered Schur form to first place the eigenvalues at zero at the upper-left corner followed by eigenvalues with negative and positive real parts, respectively. Then solving two Sylvester equations, one can obtain a matrix $Y$ to block-diagonalize $A$ as

$$
Y^{-1} A Y=\left[\begin{array}{ccc}
A_{0} & 0 & 0 \\
0 & A_{-} & 0 \\
0 & 0 & A_{+}
\end{array}\right]
$$

where the real parts of the eigenvalues of $A_{0}, A_{-}$, and $A_{+}$are zero, negative, and positive, respectively. The spectral decomposition given in (B.42) is crucial to our numerical algorithm for solving feedback fluid queues. The advantage of this decomposition is that the cases of multiple eigenvalues and/or complex eigenvalues are treated naturally without complex arithmetic and moreover we stay away from the potentially illconditioned eigenvector problem. We now present a numerical example 
to demonstrate the algorithm to obtain the decomposition (B.42) using Matlab 7.0. For this purpose, we fix

$$
A=\left[\begin{array}{ccccc}
4 & 1 & 1 / 3 & -1 & 1 / 2 \\
-1 & -4 & 1 / 3 & -1 & 1 / 2 \\
-1 & 1 & -4 / 3 & -1 & 1 / 2 \\
-1 & 1 & 1 / 3 & 4 & 1 / 2 \\
-1 & 1 & 1 / 3 & -1 & -2
\end{array}\right]
$$

which corresponds to $A=A^{(2)}=Q^{(2)}\left(R^{(2)}\right)^{-1}$ for the second regime of the five state fluid queue of Example 1. We first use the matlab function schur.m to obtain the Schur form of the matrix $A$ by issuing the command

$$
\left[Z_{1}, D_{1}\right]=\operatorname{schur}(A)
$$

which generates an orthogonal matrix $Z_{1}$ and a quasi upper-triangular matrix $D_{1}$ (simply upper-triangular for this example) such that $Z_{1}^{T} A Z_{1}=$ $D_{1}$. We obtain at this step

$$
D_{1}=\left[\begin{array}{ccccc}
5.0000 & -0.0000 & -0.0000 & 0.0000 & -0.0000 \\
0 & 1.9206 & 0.0000 & 3.5515 & -1.1180 \\
0 & 0 & -4.1758 & -1.5042 & 0.4735 \\
0 & 0 & 0 & -0.0000 & -0.6542 \\
0 & 0 & 0 & 0 & -2.0782
\end{array}\right] .
$$

We then use the matlab function ordschur.m to obtain the ordered Schur form of the matrix $A$ by issuing the command

$$
[Z, D]=\operatorname{ordschur}\left(Z 1, D 1,\left[\begin{array}{lllll}
1 & 1 & 2 & 3 & 2
\end{array}\right]\right)
$$

to place the eigenvalue at the origin (4th diagonal entry of $D_{1}$ ) to the upper-left corner and then the ones with negative real parts (3rd and 5th entries) and finally those with positive real parts (1st and 2nd). We obtain in this second step an orthogonal matrix $Z$ such that

$$
Z^{T} A Z=D=\left[\begin{array}{ccccc}
0 & -0.7155 & -0.3130 & -0.0000 & -3.3137 \\
0 & -4.1758 & -0.0511 & -0.0000 & 1.9897 \\
0 & 0 & -2.0782 & 0.0000 & 1.1073 \\
0 & 0 & 0 & 5.0000 & -0.0000 \\
0 & 0 & 0 & 0 & 1.9206
\end{array}\right]
$$


For zeroing the off-diagonal entries of the first row of $D$, we solve the Sylvester matrix equation $D(1,1) X_{1}-X_{1} D(2: 5,2: 5)=D(1,2: 5)$ or equivalently we issue the matlab command

$$
X_{1}=-D(1,2: 5) / D(2: 5,2: 5)
$$

since $D(1,1)=0$. We also zero the block off-diagonal entries in the 2 nd and 3rd rows by solving the Sylvester matrix equation $D(2: 3,2: 3) X_{2}-$ $X_{2} D(4: 5,4: 5)=D(2: 3,4: 5)$ via the matlab function lyap.m by issuing the command

$$
X_{2}=\operatorname{lyap}(-D(2: 3,2: 3), \quad D(4: 5,4: 5), \quad D(2: 3,4: 5))
$$

We finally set

$$
Y=Z\left[\begin{array}{cc}
I & -X_{1} \\
0 & I
\end{array}\right]\left[\begin{array}{ccc}
I & 0 & 0 \\
0 & I & -X_{2} \\
0 & 0 & I
\end{array}\right]
$$

which satisfies the form of (B.42) with

$$
Y^{-1} A Y=\left[\begin{array}{ccccc}
0 & 0 & 0 & 0 & 0 \\
0 & -4.1758 & -0.0511 & 0 & 0 \\
0 & 0 & -2.0782 & 0 & 0 \\
0 & 0 & 0 & 5.0000 & -0.0000 \\
0 & 0 & 0 & 0 & 1.9206
\end{array}\right] \text {. }
$$

\section{ACKNOWLEDGMENTS}

This work is supported in part by The Science and Research Council of Turkey (Tübitak) under project no. EEEAG-106E046. This manuscript has not been published elsewhere and that it has not been submitted simultaneously for publication elsewhere.

\section{REFERENCES}

1. Anick, D.; Mitra, D.; Sondhi, M.M. Stochastic theory of a data handling system with multiple sources. Bell. Syst. Tech. Jour. 1982 61, 1871-1894.

2. Tucker, R. Accurate method for analysis of a packet speech multiplexer with limited delay. IEEE Transactions on Communications 1988, 36 (4), 479-483.

3. Kulkarni, V.G. Fluid models for single buffer systems. In Frontiers in Queuing: Models and Applications in Science and Engineering; Dshalalow, J.H., Ed.; CRC Press, 1997.

4. Ramaswami, V. Matrix analytic methods for stochastic fluid flows. Proc. of the 16th International Teletraffic Congress, Smith, D., Key, P., Eds.; 1999.

5. da Silva Soares, A.; Latouche, G. Matrix-analytic methods for fluid queues with finite buffers. Perform. Eval. 2006, 63 (4), 295-314. 
6. Asmussen, S. Stationary distributions for fluid flow models with or without Brownian motion. Stochastic Models 1995, 11 (1), 21-49.

7. Rogers, L.C.G. Fluid models in queueing theory and Wiener-Hopf factorization of Markov chains. Ann. App. Prob. 1994, 4 (2), 390-413.

8. Akar, N.; Sohraby, K. Infinite and finite-buffer Markov fluid queues: A unified analysis. J. Appl. Prob. 2004, 41, 557-569.

9. Adan, I.; van Doorn, E.; Resing, J.; Scheinhardt, W. Analysis of a single-server queue interacting with a fluid reservoir. Queueing Systems 1998, 29, 313-336,

10. Scheinhardt, W. Markov Modulated and Feedback Fluid Queues. Ph.D. dissertation, University of Twente, 1998.

11. Elwalid, A.; Mitra, D. Statistical multiplexing with loss priorities in rate-based congestion control of high-speed networks. IEEE Trans. Commun. 1994, 42, 2989-3002.

12. Kella, O.; Stadje, W. Exact results for a fluid model with state-dependent flow rates. Prob. Eng. Informat. Sci. 2002, 16 (4), 389-402.

13. Scheinhardt, W.; Foreest, N.; Mandjes, M. Continuous feedback fluid queues. Oper. Res. Lett. 2005, 33, 551-559.

14. Mandjes, M.; Mitra, D.; Scheinhardt, W. Models of network access using feedback fluid queues. Queueing Syst. Theory Appl. 2003, 44 (4), 2989-3002.

15. Mandjes, M.; Mitra, D.; Scheinhardt, W. A simple model of network access: feedback adaptation of rates and admission control. Computer Networks 2003, 41, 489-504.

16. Boxma, O.; Kaspi, H.; Kella, O.; Perry, D. On-off storage systems with state-dependent input, output, and switching rates. Probab. Eng. Inf. Sci. 2005, 19 (1), 1-14.

17. Kankaya, H.E.; Akar, N. Performance Evaluation and Traffic Modeling Tools. http://www.ee.bilkent.edu.tr/ pevatools/, Electrical and Electronics Engineering Department, Bilkent University.

18. Golub, G.H.; van Loan, C.F. Matrix Computations, 3rd Ed.; The Johns Hopkins University Press, 1996.

19. LAPACK Users's Guide, 1995, 2nd Ed.

20. MATLAB 7.0.0.19901 (R14), The MATH WORKS Inc., 2005.

21. van Dooren, P.M. Numerical linear algebra for signals, systems, and control. Universite Catholique de Louvain, Belgium. 2003. Draft notes prepared for the Graduate School in Systems and Control.

22. Bai, Z.; Demmel, J.; Gu, M. Inverse free parallel spectral divide and conquer algorithms for nonsymmetric eigenproblems. Numer. Math. 1997, 76, 389-396. 